\title{
Superselective ophthalmic artery fibrinolytic therapy for the treatment of central retinal vein occlusion
}

M Paques, J N Vallée, D Herbreteau, A Aymart, P Y Santiago, F Campinchi-Tardy, D Payen, J J Merlan, A Gaudric, P Massin

\begin{abstract}
Aim-To study the effect of superselective ophthalmic artery fibrinolysis as a treatment for central retinal vein occlusion (CRVO).

Methods-Retrospective, university based single centre study. The charts of 26 eyes of 26 patients treated were reviewed. Among the 26 patients, there were nine cases of combined artery and vein occlusion, three cases of combined cilioretinal artery and CRVO, and 14 cases of classic CRVO. Complete preoperative and postoperative ophthalmological examination and fluorescein angiography were performed in all cases. The therapeutic procedure comprised the infusion of urokinase through a microcatheter into the ostium of the ophthalmic artery, via a femoral artery approach. The main outcome measure was the improvement in visual acuity 48 hours after the procedure. Results-Six eyes of six patients exhibited significant improvement in visual acuity immediately after the fibrinolysis procedure. Among them, four had a initial funduscopic appearance suggestive of combined occlusion of the central retinal artery (CRAO) and vein. For these patients, the visual benefit was maintained in the long term. Intravitreal haemorrhage occurred in two patients. There were no extraocular complications linked to the procedure.

Conclusions-Selective ophthalmic artery infusion of urokinase was followed by improvement in VA in six out of 26 cases of CRVO. Eyes with combined CRAO and CRVO with recent visual loss appeared to be the most responsive. This treatment did not prevent the occurrence of ischaemia in the failure cases. The efficacy of in situ fibrinolysis for treatment of CRVO needs to be further evaluated in a controlled study.

(Br f Ophthalmol 2000;84:1387-1391)
\end{abstract}

Central retinal vein occlusion (CRVO) is a common cause of visual loss. ${ }^{1} \mathrm{CRVO}$ is considered to be consecutive to an obstruction of the retinal venous circulation, causing dilatation of retinal veins and retinal haemorrhages. Secondary complications include macular oedema and widespread capillary non-perfusion, the latter possibly leading to neovascular glaucoma. The presence at the initial stage of a thrombus in the central vein have been suggested by histopathological studies. ${ }^{2}$ The involvement of other factors such as increased blood viscosity, elevated red blood cell aggregability, central vein compression by the central retinal artery is debated. ${ }^{3}$ The treatment of CRVO remains controversial and overall disappointing. ${ }^{4}$

Peripheral venous administration of fibrinolytic therapy has been proposed, but its risk/benefit ratio is uncertain. ${ }^{5}$ Recent progress in interventional neuroradiology has made possible the selective catheterisation of intracranial small vessels such as the ophthalmic artery. ${ }^{6}$ Therefore, to maximise the concentration of fibrinolytic agent at the obstruction site and reduce the risk of systemic fibrinolysis complications we treated patients with CRVO by selective infusion of urokinase into the ophthalmic artery. We report the results of this pilot study.

\section{Methods}

The charts of all patients who underwent superselective ophthalmic artery fibrinolysis for CRVO in our department were reviewed for this retrospective study. This treatment was proposed when initial visual acuity was below 20/60, and/or if there has been CRVO in the fellow eye, and/or if worsening of VA occurred after initial improvement and/if the other eye was lost. The usual contraindications for intracerebral catheterisation were complied with-that is, patient had not undergone recent surgery, myocardial infarction, or stroke, and did not have uncontrolled hypertension.

\section{PREOPERATIVE EXAMINATION}

Each patient had a complete ophthalmological examination by one of us (PM, MP, or AG) including recording of their ophthalmological history, date of onset of visual symptoms, best corrected visual acuity (VA) on a decimal chart, biomicroscopy of the anterior segment, applanation tonometry and fundus examination. CRVO was diagnosed on the basis of the presence of retinal vein dilatation in four quadrants, combined with retinal haemorrhages. A fluorescein angiography was performed with emphasis on early phase frames in order to evaluate blood velocity in the retinal vessels.

Each patient gave informed consent to the treatment. 


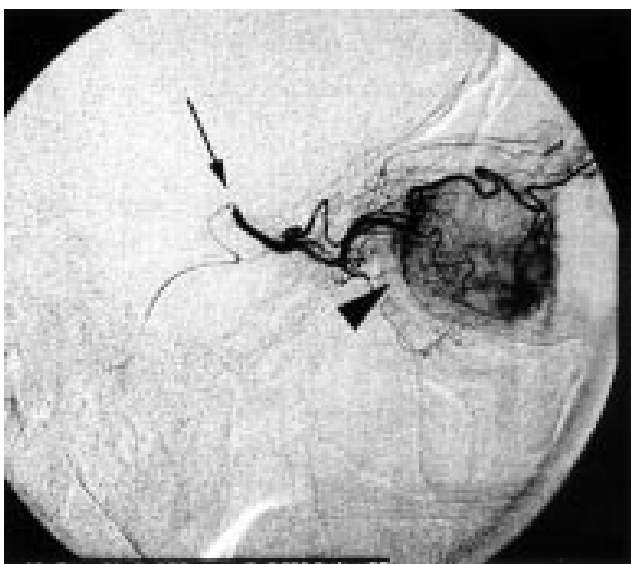

Figure 1 Superselective ophthalmic artery angiogram performed immediately before infusion of urokinase showing the choroidal blush (arrowhead). The arrow indicates the tip of the microcatheter in the ostium of the ophthalmic artery.

THERAPEUTIC PROCEDURE

Treatment was performed in the neuroradiology department under local anaesthesia by a neuroradiologist trained in the catheterisation of intracranial vessels. Briefly, the internal carotid artery was catheterised via a femoral artery approach with a 5 Fr guide catheter, and a 0.035 inch hydrophylic coated guidewire (Terumo, Japan). An internal carotid cerebral angiogram was performed to identify the carotid artery siphon. The $5 \mathrm{Fr}$ guide catheter was positioned inside the proximal extracranial part of the internal carotid artery. A 1.8 Fr or 1.5 Fr flow guide microcatheter (Magic, Balt Extrusion, France; Spinneker, Boston Scientific, MA, USA) was advanced through the guide catheter into the ostium of the ophthalmic artery. A superselective ophthalmic angiogram (Fig 1) was performed to assess the correct placement of the tip of the microcatheter in the ophthalmic artery ostium. Urokinase (300 000 IU prediluted with $0.9 \%$ normal saline; Hoechst, France) was then perfused for 40 minutes through the microcatheter at a continuous flow rate using an electric pump. This required overall 1 hour of operating management.

FOLLOW UP

As strict bedrest was maintained between 12 and 24 hours after the procedure, VA was not measured until the next day or day 2. At each follow up visit, a complete ophthalmological examination were performed in our department.

\section{Results}

Patients' clinical characteristics before treatment are given in Table 1. The series comprised 19 men and seven women. Their mean age was 61 years (range 42-85). Eleven patients ( $42 \%$ ) had VA below $20 / 200$ vision in the fellow eye (due to CRVO in nine cases and trauma in two). Ten patients (38\%) had glaucoma and/or elevated intraocular pressure in the same or the fellow eye. Thirteen (50\%) were treated for hypertension. Before fibrinolysis treatment, the mean duration of visual symptoms was 26 days (range 12 hours-7 months; median, 5 days). Seventeen patients had loss of vision lasting for less than 14 days. Thirteen patients $(46 \%)$ had a VA of counting fingers or less. Loss of vision was believed to be due to macular oedema in 12 cases, to impairment of central retinal artery perfusion in nine cases, to cilioretinal artery occlusion in three cases, to macular haemorrhage in one case, and to macular ischaemia in one case.

\section{PEROPERATIVE INCIDENTS}

In one patient, catheterisation of the ophthalmic artery ostium was not possible owing to the high degree of vascular tortuosity, and therefore urokinase was injected proximally to the ostium but not in the ostium itself.

Table 1 Clinical characteristics of 26 patients with central retinal vein occlusion before fibrinolysis treatment

\begin{tabular}{|c|c|c|c|c|c|c|c|c|c|c|}
\hline Case & Age & Sex & $\begin{array}{l}\text { Glaucomal } \\
\text { IOP } \\
>21 \mathrm{~mm} \mathrm{Hg}\end{array}$ & $\begin{array}{l}\text { Duration of } \\
\text { visual loss } \\
\text { (days) }\end{array}$ & Initial VA & $\begin{array}{l}\text { Cause of } \\
\text { visual loss }\end{array}$ & $\begin{array}{l}\text { VA at } \\
\text { day } 1\end{array}$ & Last VA & $\begin{array}{l}\text { Rubeosis/ } \\
\text { NVG }\end{array}$ & $\begin{array}{l}\text { Follow up } \\
\text { (months) }\end{array}$ \\
\hline 1 & 66 & $\mathrm{~F}$ & Yes & 0.5 & $\mathrm{CF}$ & IBF & $20 / 40$ & $20 / 50$ & - & 16 \\
\hline 2 & 53 & $M$ & - & 1 & $20 / 400$ & IBF & $20 / 50$ & $20 / 20$ & - & 6 \\
\hline 3 & 45 & $M$ & - & 0.5 & $\mathrm{CF}$ & IBF & $20 / 20$ & $20 / 20$ & - & 5 \\
\hline 4 & 67 & $M$ & Yes & 2 & $20 / 160$ & IBF & $20 / 100$ & $20 / 50$ & - & 4 \\
\hline 5 & 59 & $M$ & - & 11 & $\mathrm{CF}$ & IBF & $\mathrm{CF}$ & CF & Rubeosis & 28 \\
\hline 6 & 44 & M & - & 2 & $\mathrm{CF}$ & IBF & $\mathrm{CF}$ & CF & - & 3 \\
\hline 7 & 58 & $\mathrm{~F}$ & - & 0.5 & $\mathrm{CF}$ & IBF & $\mathrm{CF}$ & $20 / 200$ & - & 3 \\
\hline 8 & 51 & $M$ & - & 2 & $\mathrm{CF}$ & IBF & $\mathrm{CF}$ & $20 / 200$ & - & 3 \\
\hline 9 & 56 & $\mathrm{~F}$ & Yes & 0.5 & $\mathrm{CF}$ & IBF & LP & LP & - & 6 \\
\hline 10 & 42 & $M$ & - & 30 & $20 / 50$ & MO & $20 / 25$ & $20 / 200$ & - & 22 \\
\hline 11 & 69 & $M$ & - & 2 & $\mathrm{CF}$ & MO & $\mathrm{CF}$ & $\mathrm{CF}$ & Rubeosis & 15 \\
\hline 12 & 45 & $\mathrm{~F}$ & - & 4 & $\mathrm{CF}$ & MO & $20 / 200$ & $20 / 20$ & - & 14 \\
\hline 13 & 85 & M & - & 3 & $\mathrm{CF}$ & MO & HM & $\mathrm{HM}$ & - & 2 \\
\hline 14 & 65 & $M$ & - & 5 & $20 / 100$ & MO & $20 / 100$ & $20 / 200$ & - & 7 \\
\hline 15 & 75 & $\mathrm{~F}$ & - & 120 & $\mathrm{CF}$ & MO & $\mathrm{CF}$ & $20 / 400$ & - & 32 \\
\hline 16 & 58 & M & - & 210 & $20 / 50$ & $\mathrm{MO}$ & $20 / 50$ & $20 / 60$ & - & 53 \\
\hline 17 & 61 & $\mathrm{M}$ & Yes & 30 & $20 / 50$ & MO & $20 / 40$ & $\mathrm{CF}$ & - & 18 \\
\hline 18 & 56 & M & - & 60 & $20 / 100$ & MO & $20 / 100$ & $20 / 100$ & - & 71 \\
\hline 19 & 57 & M & Yes & 90 & $20 / 50$ & MO & $20 / 50$ & $20 / 400$ & NVG & 31 \\
\hline 20 & 65 & $M$ & - & 60 & $20 / 400$ & MO & $20 / 400$ & $20 / 400$ & & 4 \\
\hline 21 & 69 & $\mathrm{~F}$ & Yes & 5 & $20 / 50$ & MO & $20 / 40$ & $20 / 25$ & & 6 \\
\hline 22 & 52 & $M$ & - & 1 & $20 / 60$ & CR & $20 / 25$ & $20 / 25$ & & 34 \\
\hline 23 & 73 & $M$ & Yes & 18 & $20 / 25$ & CR & $20 / 40$ & $20 / 20$ & & 32 \\
\hline 24 & 60 & $\mathrm{M}$ & - & 7 & $20 / 160$ & CR & $20 / 160$ & $20 / 200$ & & 4 \\
\hline 25 & 50 & $M$ & Yes & 2 & $\mathrm{CF}$ & $\mathrm{SMH}$ & $\mathrm{CF}$ & $\mathrm{HM}$ & Rubeosis & 3 \\
\hline 26 & 73 & $\mathrm{~F}$ & - & 15 & $\mathrm{CF}$ & Ischaemia & HM & $\mathrm{HM}$ & NVG & 32 \\
\hline
\end{tabular}

$\mathrm{NVG}=$ neovascular glaucoma; $\mathrm{IBF}=$ impairment of retinal blood flow; $\mathrm{MO}=$ macular oedema $\mathrm{CR}=$ cilioretinal artery occlusion; $\mathrm{SMH}=$ submacular haemorrhage. 

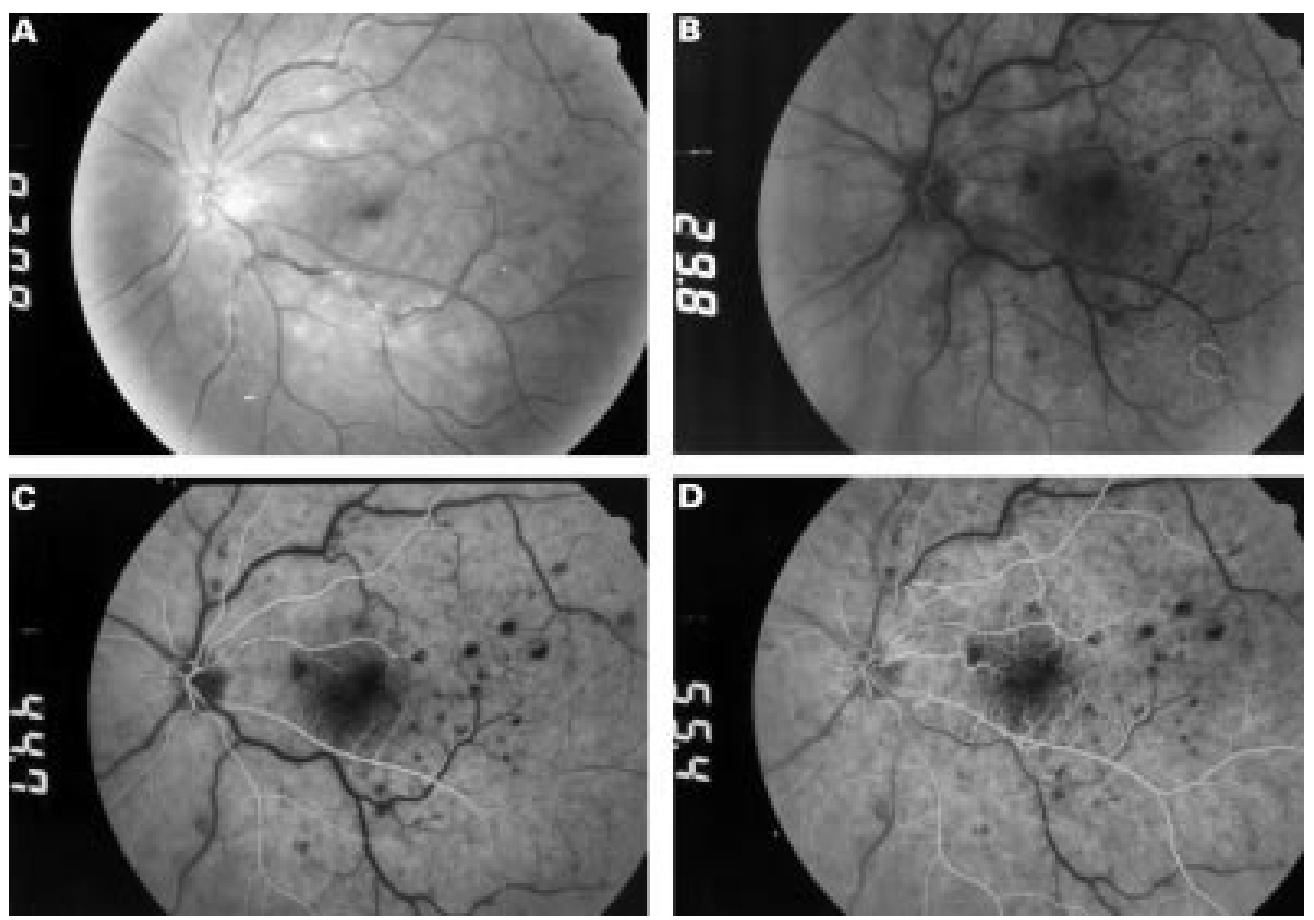

Figure 2 Case 2. Preoperative features. VA was 20/400. (A) Preoperative fundus photograph showing scattered retinal haemorrhages, venous dilatation and tortuosity, cotton wool spots, and areas of inner retinal ischaemia at the posterior pole. (B) Early frame of preoperative fluorescein angiography showing filling of the central retinal artery. (C) Fifteen seconds later, the retinal arteries are not completely filled indicating severely impaired retinal arterial supply. (D) Arteriovenous phase of fluorescein angiography showing that there is no capillary closure at the posterior pole.

However, a cerebral angiogram showed that most of the infused urokinase was directed in the ophthalmic artery. During the catheterisation procedure, none of the patients developed neurological symptoms, and no extraocular side effects were noted after fibrinolysis.

FUNCTIONAL RESULTS

Six patients (cases 1, 2, 3, 4, 11, and 22) noticed subjective improvement in VA within hours of the procedure, and at 48 hours objective VA was found to have improved significantly. In two of these patients (cases 2 and 3) retinal perfusion appeared markedly improved on fluorescein angiography (Figs 2 and 3). This improvement was maintained in the long term in five patients. Two others noticed a prompt improvement in their visual field, but a residual central scotoma persisted probably explaining why objective VA did not improve. In case 2 examination was performed 6 months after fibrinolysis and showed 20/20 VA and a normal fundus (Fig 4).

Among the nine patients with combined artery and vein occlusion, four had an improvement after the procedure. Among the three patients with ciliretinal artery occlusion combined to CRVO, one showed improvement. Among the other cases, only one showed improvement but CRVO recurred and final VA was poor.

Three patients (cases 7, 21, and 25) had a second in situ fibrinolysis procedure during the week after the first unsuccessful one. None of the patients retreated had significant visual improvement. Mean follow up was 10 months (range 2-24). Pre- and post-treatment data are given in Table 1.
FUNDUSCOPIC APPEARANCE

In eight cases, the number of retinal haemorrhages increased the day after the procedure, as the case shown in Figure 5.

Two patients (cases 9 and 13) had an intravitreal haemorrhage in the immediate postoperative period, that necessitated vitrectomy in both cases. Final VA was poor.

The patients with visual improvement exhibited progressive clearing of the haemorrhages and normalisation of retinal veins over a few weeks.

Neovascular glaucoma developed in four patients, who did not experience improvement in VA after treatment, and rubeosis iridis without elevated intraocular pressure developed in one. Prompt photocoagulation was performed in these cases, and was followed by the regression of neovascularisation. These five patients all had final vision of light perception, intraocular pressure was controlled by topical medication, and none of them underwent enucleation.

\section{Discussion}

Six of our 26 patients experienced a striking improvement in their VA in the 24 hours after fibrinolysis. When analysing the pretherapeutic data for the six patients who responded to treatment, we found that four of them (cases 1-4) had a funduscopic appearance suggestive of combined central retinal artery occlusion (CRAO) and CRVO, defined by the association of venous dilatation, retinal haemorrhages, and areas of retinal inner ischaemia at the posterior pole. ${ }^{7}$ On fluorescein angiography of these four patients, retinal arterial perfusion was markedly impaired, but none had capillary 

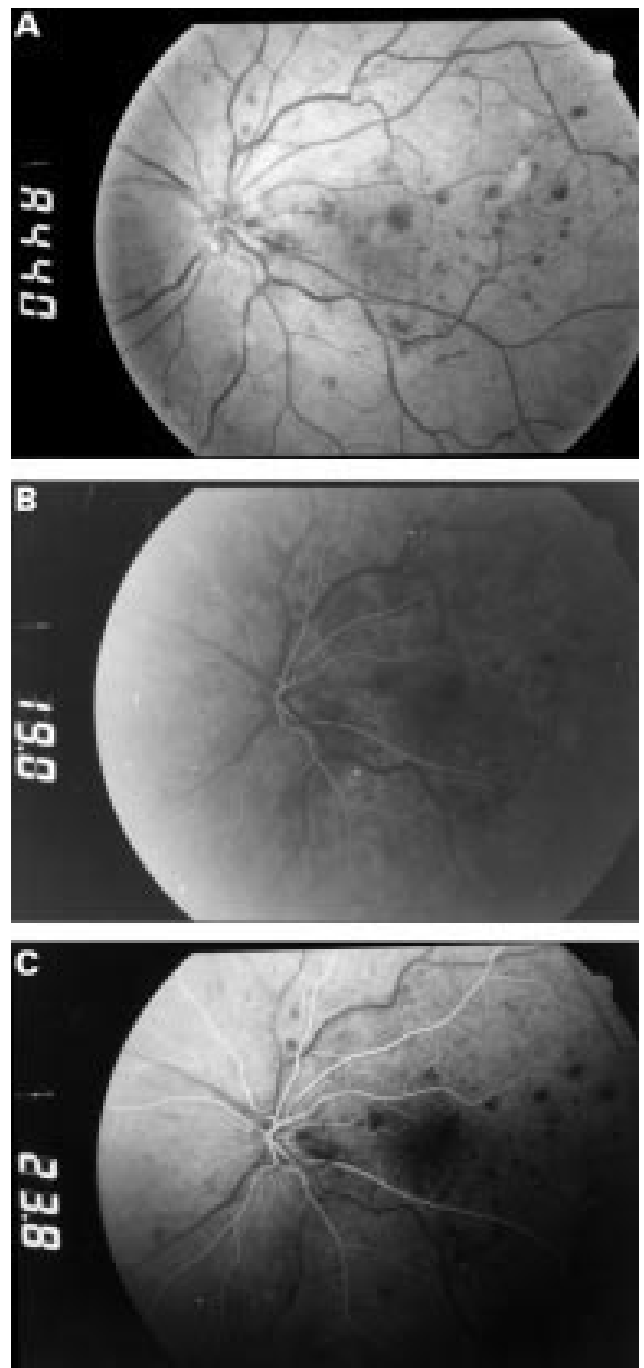

Figure 3 Case 2, the day after in situ fibrinolysis. VA was 20/50. (A) Red-free photograph showing a few more scattered haemorrhages than preoperatively. (B) Early frame of fuorescein angiogram showing that the filling of retinal arteries is beginning shortly after choroidal filling. (C) The beginning of the arteriovenous transit time occurs approximately 4 seconds after the appearance of the dye in retinal arteries, indicating an improvement in retinal circulation times.

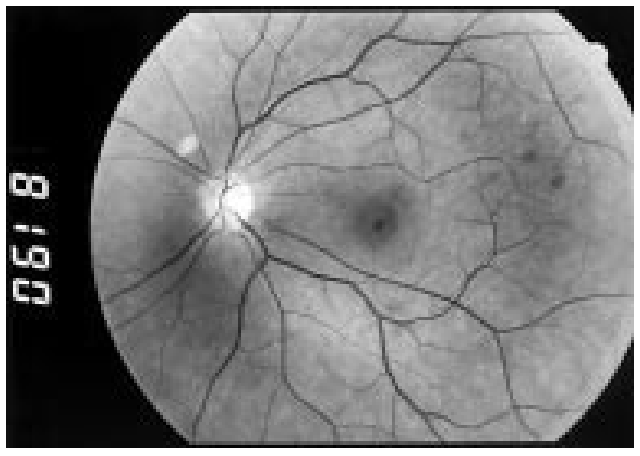

Figure 4 Case 2. Fundus appearance 6 months later. VA was 20/20. There is mild residual narrowing of retinal vessels.

closure or macular oedema. Therefore we believe that in these patients, visual loss was due to the extreme slowing of retinal arterial perfusion. Among all patients, nine patients (cases 1-9) had a funduscopic appearance of
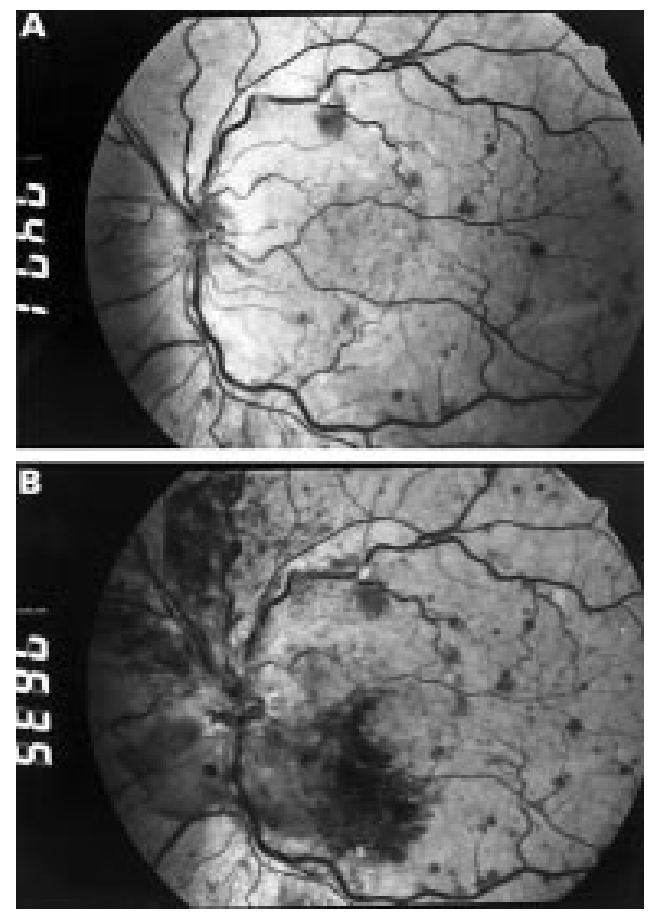

Figure 5 Case 6. (A) Preoperative red-free photograph. (B) Immediate post-fibrinolysis fundus photograph. Note the increased number of haemorrhages.

combined CRAO and CRVO, with initial visual acuity ranging from counting fingers to $20 / 160$. Four improved, and their final VA was $20 / 50$ or better. The other five had a final VA of 20/200 or less. Since most of the reported cases of combined CRVO and CRAO have had a poor visual outcome, with a high frequency of rubeosis, a beneficial effect of in situ fibrinolysis in this subset of CRVO is possible. However, our study lacks a control group, and spontaneous improvement of combined CRAO and CRVO have also been reported..$^{8-10}$ In our series, the high proportion of patients with combined CRAO and CRVO (9/26, 35\%) indicates that it may be more frequent than was previously believed, and that it may constitute an initial form of "classic" CRVO. Indeed two patients (cases 6 and 8) with combined CRVO and CRAO at presentation eventually developed numerous retinal haemorrhages and macular oedema.

For the other patients, the relation between the treatment, circulatory changes, and visual results was not clear in all cases. For instance, case 10, whose VA also improved, had numerous retinal haemorrhages and macular oedema. His VA improved from $20 / 50$ to $20 / 30$ immediately after fibrinolysis, despite the absence of visible changes in clinical and angiographic features. One month later he again had loss of vision to 20/200.

Overall, among the 15 patients with initial VA below 20/200, 11 (73\%) had final VA of $20 / 200$ or less, and three of the six patients (50\%) with initial VA of $20 / 50$ or more had a comparable final VA. These data are similar to the results of the CRVO study. ${ }^{11}$

Fibrinolysis therapy by peripheral intravenous infusion of urokinase has been proposed for CRVO. Kohner et al reported in a 
randomised controlled trial that intravenous streptokinase did not have a clear cut effect on visual acuity. ${ }^{5}$ Conversely, in the study reported by Elman, ${ }^{12}$ who treated CRVO patients by intravenous infusion of tissue plasminogen activator in a non-randomised trial, 57 out of 96 patients (59\%) with initial VA below 20/100 gained three or more lines of vision at 6 months, which appears to be better than in the report from the CRVO study.

The main risk of fibrinolytic therapy is haemorrhage. Kohner et al reported that three CRVO patients out of 20 had intravitreal haemorrhage, and three of 96 patients in the study by Elman. One included in the former died of stroke. In order to avoid general haemorrhagic accidents, lower doses of fibrinolytic agent ${ }^{13}$ or in situ infusion ${ }^{6}{ }^{14}$ can be used. Intravitreal haemorrhage, which occurred in two of our cases, may be considered a serious problem if it delays the diagnosis of retinal ischaemia. Vitrectomy may therefore be indicated for these eyes if the vitreous does not clear rapidly. Specific to intracranial vessel catheterisation is the risk of postoperative neurological deficit. Consequently, this procedure should not be used for patients with a history of stroke, or in the presence of extensive atherosclerotic lesions.

The benefit of this treatments need to be evaluated in a prospective controlled study with strict inclusion criteria. Our data suggest that only patients with duration of vision loss of less than 8 days, without macular oedema should be included in such a study. The analysis of retinal circulatory dynamics should also be taken into account, since arterial flow impairment was associated with a favourable prognosis.

Presented in part at the meeting of the Association for Research in Vision and Orthere merch 1996.

The authors have no proprietary interest in the present study.

1 Mitchell P, Smith W, Chang A. Prevalence and associations of retinal vein occlusion in Australia. The Blue Mountains of retinal vein occlusion in Australia. The Blue

2 Green WR, Chan CC, Hutchins GM, et al. Central retinal vein occlusion. A prospective histopathological study of 29 eyes in 28 cases. Retina 1981;1:27-55

3 Rath EZ, Frank RN, Schin DH, et al. Risk factors for retinal vein occlusions. Ophthalmology 1992;99:509-14.

4 Laatikainen L. Management of retinal vein occlusion. Curr Opin Ophthalmol 1992;3:372-8.

5 Kohner EM, Pettit JE, Hamilton AM, et al. Streptokinase in central retinal vein occlusion : a controlled clinical trial. BFM 1976;1:550-3.

6 Zeumer H, Freitag HJ, Zanella F, et al. Local intra-arterial fibrinolytic therapy in patients with stroke: Urokinase versus recombinant tissue plasminogen activator (rt-PA). versus recombinant tissue plasm
Neuroradiology 1993;35:159-62.

7 Brown G, Duker J, Lehman R, et al. Combined central retinal artery central vein obstruction. Int Ophthalmol 1993;17: 9-17.

8 Jorizzo PA, Klein ML, Shults WT, et al. Visual recovery in combined central artery and central vein occlusion. $A m$ Ophthalmol 1987;104:358-63.

9 Iijima $\mathrm{H}$, Tsumara T. Combined occlusion of the central retinal artery and vein. fpn f Ophthamol 1994;38:202-7.

10 Keyser BJ, Duker JS, Brown GC, et al. Combined central retinal vein occlusion and cilioretinal artery occlusion assoretinal vein occlusion and cilioretinal artery occlusion asso-
ciated with prolonged retinal arterial filling. Am 7 Ophthalmol 1994;117:308-13.

11 The Central Vein Occlusion Study Group. Natural history and clinical management of central retinal vein occlusion. Arch Ophthalmol 1997;115:486-91.

12 Elman MJ. Thrombolytic therapy for central retinal vein occlusion: results of a pilot study. Trans Am Ophthalmol Soc 1996;94:471-504

13 Hattenbach LO, Steinkamp G, Scharrer I, et al. Fibrinolytic therapy with low-dose recombinant tissue plasminogen activator in retinal vein occlusion. Ophthalmologica 1998; 212:394-8.

14 Schmidt D, Schumacher M, Wakhloo AK. Microcatheter urokinase infusion in central retinal artery occlusion. $A m \mathcal{F}$ Ophthalmol 1992;113:429-34. 